\title{
Real-Time Ballot Box Monitoring System
}

\author{
Bamidele Moses Kuboye \\ Department of Information Technology, \\ Federal University of Technology, Akure, \\ P. M. B. 704, Akure, Ondo State, Nigeria
}

\author{
Akintoba Emmanuel Akinwonmi \\ Department of Computer Science, \\ Federal University of Technology, Akure, \\ P. M. B. 704, Akure, Ondo State, Nigeria
}

\begin{abstract}
A Real-time Ballot Box Monitoring is a system which tracks and monitors the location of ballot box during the voting process in real-time with GSM and GPS sensors. In this work a tracking device using Seeeduinomicro-controller and embedded software was developed. A monitoring station where the ballot box is constantly monitored online was also developed using web application. It will enable ballot papers and important electoral materials to be better monitored, hence making election results more credible and will reduce fraudulent rigging and manipulation of election process in Nigeria.
\end{abstract}

\section{General Terms}

Internet of Things (IoT), Sensor, Election

\section{Keywords}

Ballot, Global Positioning System (GPS), Long Term Evolution (LTE), Global System for Mobile Communication (GSM), Network

\section{INTRODUCTION}

The Internet of Things (IoT) is the inter-connection and exchange of data among devices or sensors [1]. An IoT is a type of a wireless sensor networks (WSN) that comprises of a set of embedded processing units or sensors communicating via wireless links [2]. Additionally, IoT objects are embedded with tiny computers that can monitor surroundings, display information, and perform actions with some degree of autonomy. Ashton used the IoT for the first time in 2009 to define radio-frequency identifications with internet connectivity to enhance supply chain logistics [3]. The main function is the collection of parameters related to the surrounding environment, such as temperature, pressure, the presence or movement of objects[2].

The ever increasing growth of the IoT technologies has brought about a growing number of practical applications in many fields including security, asset tracking, agriculture, smart metering, smart cities, and smart[1]. Embracement of IoT technologies by individuals and organizations had led to a well suited management of large numbers of assets and distributed processes [4]. Applications of IOT have proved to be useful in many areas such as home, health, transport, offices, communities, nations. Application of IOT is of great benefits to national developments. Nigeria is a nation with different culture with intersecting regional, religious, and ethnic boundaries. Nigeria present population estimates by World Bank population data presentation is around 200 million [5]. A new dispensation of the present Democracy started in 1999 after over thirty (30) years of military rule with the elections into the presidency, the national Assembly, governorship and State Houses of Assembly in the 36 states.
Though voting is the fundamental right in any democracy, however, the process of this voting has been compromised in many occasions due to violence, preferential treatment and so on. Recently as reported by Vanguard newspaper in Nigeria that the INEC's Resident Electoral Commissioner (REC) in Akwalbom said that three university lecturers had been confirmed to have been involved in electoral malpractice during the 2019 general elections [6].

In other to protect the integrity of elections, different methods have been employed right from physical counting to the Internet voting. The Independent National Electoral Commission (INEC) introduced the Permanent Voters Card (PVC) card reader for electronic authentication of voters as one of the measures to curb electoral malpractices. The card reader was used for the first time in Nigeria on March 28, 2015 during the presidential election [7]. As at present, PVC functions has not been fully harnessed because there has not been a full blowing central database to authenticate the voters in other to curb malpractices. A lack of ballot boxes integrity protection during elections could lead to ballot stuffing and other unscrupulous practices which will amount to electoral fraud. Presently, ballot box is monitored physically through manual process by the electorates, security officers, election observers and INEC staffs. However, this method has failed to instantaneously inform the appropriate quarters in situations of ballot box snatching or tampering with the content of the ballot box. Hence, it would be more advantageous if the tampering or snatching is detected immediately and the location being tracked in real-time. With the advent and improvement in Internet of Things (IoT) technology, this can be done, thereby, reduced fraud to barest minimum.

In the light of this, a system is developed to monitor ballot box during the electioneering process. This system should be able to reduce ballot box snatching to the barest minimum. So this work comes up with a system that will detect the event arising from ballot box snatching through the proposed Ballot Box Tracking and Monitoring System. The ballot box tracking system will use GPS and GSM to provide data for the monitoring of the ballot box location in real-time and provide better control. The rest of the paper is organized thus; the IOT brief layout and related works are discussed in section 2 and 3 while section 4 and 5 gave the concept and implementation of the work and section 6 gives the conclusion.

\section{INTERNET OF THINGS (IoT)}

Internet of things (IoT) is considered as a network of physical things which are connected through Internet and able to generate, extract, and record data for real-time monitoring and decision-making in a variety of applications [8];[9]. Lawal and Rasfsanjani[10] defined IoT as a system made up of internet-enabled objects such as sensors and devices that are connected over internet and able to transfer data over wireless 
networks to remotely communicate with each other. In addition, IoT literature has introduced several IoT systems architecture, but a general architecture of an IoT system consists of physical, cloud, communication, and service layer was presented by Lawal and Rasfsanjani, [10] in Fig. 1. The physical layer consists of the different sensors, actuators, processors, and other physical devices that are connected intelligently to each other to communicate with the environment. The sensors monitor and respond to events in the environments. The cloud layer consists of technologies such as computing, database, and big data processing. Data collected by the sensor is stored, analyzed with devised algorithms to make decisions and afterwards sent to the service layer for visualization. The communication layer is the network layer that is in control of the connectivity of the IoT system through network technologies to the other layers for secured and complete data transfer [10]. This layer connects all the computing devices in the location to the internet and allows them to communicate via Internet Protocol (IP) [11]. In the cellular communication, over the years, network operators have been enhancing their infrastructure to support data capability and improving network throughput for their existing cell sites, transceivers, and interconnection facilities.

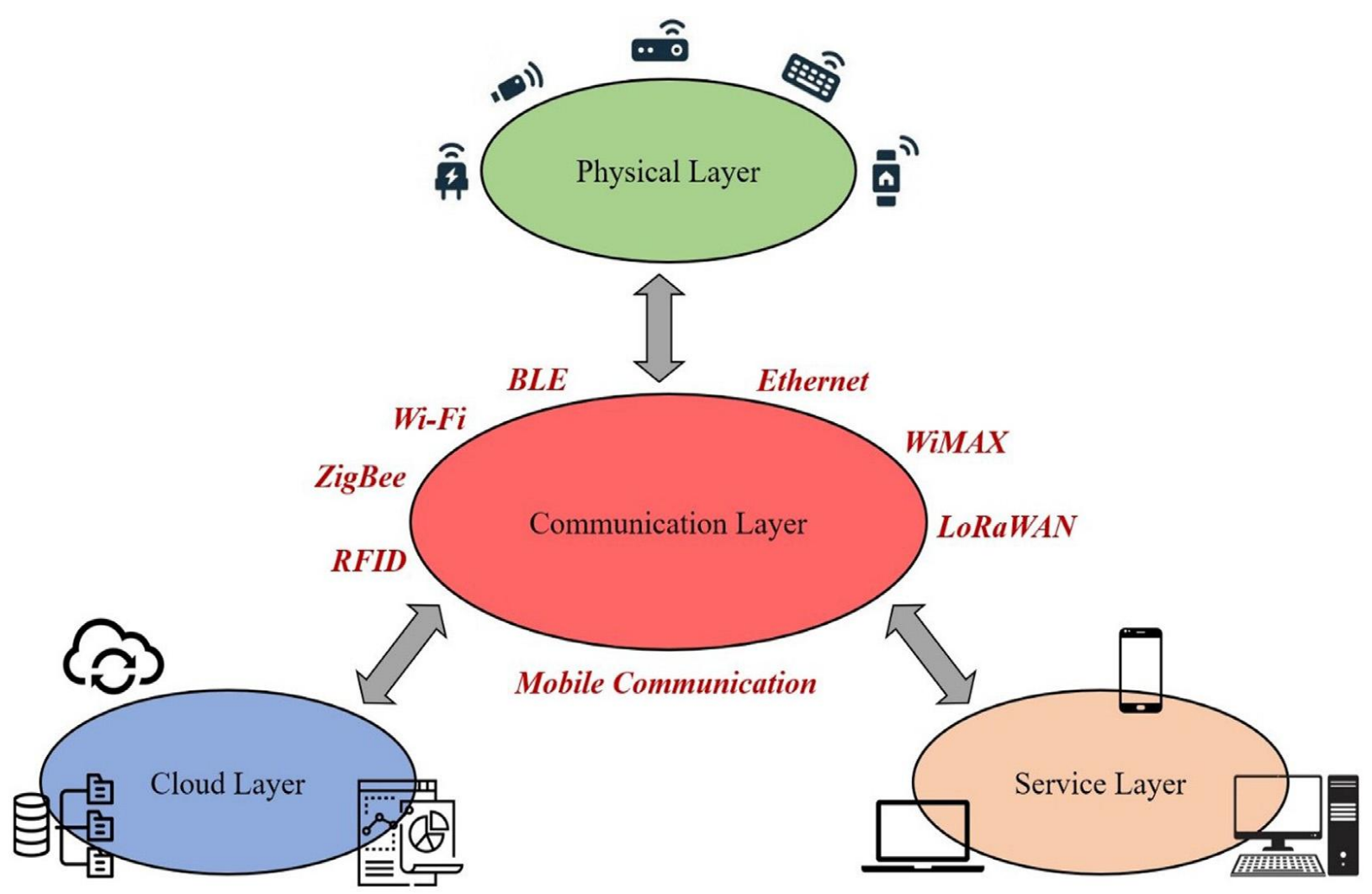

Fig 1: IoT system architecture [10]

As the technology trend shifts towards providing faster data rates and lower latency connectivity, the Third Generation Partnership Project (3GPP) standards body has developed radio platform called Long Term Evolution (LTE). LTE offers a number of distinct advantages such as increased performance attribute, high peak data rates, low latency and greater efficiencies in using the wireless spectrum [12]. Low latency makes it possible for IoT applications to query or receive quicker updates from sensor devices [13]. Aside the 3GPP platform, some of the other communication technologies that can be used for monitoring include Ethernet, Wi-Fi, WiMAX, Zigbee, mobile communications, Long Range Wide Area Network (LoRaWAN), Radio Frequency Identification (RFID), and Bluetooth Low Energy (BLE) [10]. The communication between IoT devices is mainly wireless because they are generally installed at geographically dispersed locations [11]. A cloud layer allows data collected by the sensor to be stored and analyzed with various algorithms to make decisions. Thereafter, the decision made will be sent to the service layer for visualization [10]. Cloud computing is a model for on-demand access to a shared pool of configurable resources such as computers, networks, servers, storage, applications, services, software and so on[14]. Cloud computers allows large centralized processing of data, thus, provides great flexibility and scalability[11]. It deals with services such as the core infrastructure, platform, software, and storage. The service layer functions as a link between the users and the IoT system [15]. This layer consists of web and mobile terminal that allows users to view the processed data in form of graphs, charts, or spreadsheets. It offers remote controls various devices connected in the IoT system.

\section{REVIEW OF RELATED LITERATURE}

Sensors help to collect data from the environment, thus, it is an essential components of smart objects. The Global Positioning System (GPS) as one of the IOT sensors is mainly

for detection of location of an attached object, which is one of the most important pieces of contextual information for smart applications. The GPS distance is measured from three or more satellites and coordinates are computed [11]. Wireless sensor based remote monitoring system for agriculture using 
zigbee and GPS was developed by Satyanarayana et al[16]. The system developed a wireless sensor network to help farmers in evaluating soil conditions and act accordingly. The system obtains Global Positioning System (GPS) parameters related to the field and sends them to a central monitoring station. The system consists of wireless sensor network nodes and network management platform. Zigbee transmit acquisition of the temperature and humidity data to the Zigbee stations of gateways node which then transmits the data to GPRS PC for real-time data view. The system was able to monitor the environmental temperature and humidity of the soil. The research was only limited to Agriculture.

Rathinakumar and Manivannan[17] build a wireless accident information system using GSM and GPS. The main focus of the system is to provide security for the vehicle user as well as detects the accident if occurred and informs the respective authority through wireless technologies. The system used three modules, automatic speed control module, accident detection and information sending module as well as security enabling module. In automatic speed control module, Radio Frequency (RF) nodes are used and they consist of RF transmitter and RF receiver. The RF transmitter is placed in a specific location and RF receiver is placed in vehicle. When the vehicle reaches the zone like school, round-about turn, it will automatically reduce the speed and when it leaves the zone it will automatically regain its speed. In information sending module GSM, GPS and vibration sensors are used. In this system, the vibration sensor, GPS and GSM are placed in the vehicle. If an accident occurred the vibration sensor senses the vibration level and if it exceeds the threshold limit, the system will consider that there is an occurrence of accident in that particular location. Then, the system will activate the GPS to gather the location detail and sends the location of the vehicle through GSM to the control station. Security enabling module includes eye sensor and seat belt detector which will ensure the security condition of the driver. This study tends to provide a realistic measure to the issues such as automatic speed control mechanism, accident detection and information sending, thereby, reduces the accidents and save the human lives if implemented.

Real time vehicle tracking system using GSM and GPS technology was studied by [18]. This paper proposed a vehicle tracking system that works using GPS and GSM technology. The AT89C51 microcontroller was used to interface various hardware peripherals. A GSM modem is used to send the position data, that is, Latitude and Longitude, of the vehicle given by the GPS from a remote place. The GPS modem will continuously give the position data of the vehicle and anytime a request by user is sent to the mobile, the system automatically sends a return reply to that mobile indicating the position of the vehicle. The system was designed and implemented for an anti-graft system but can be used for vehicle monitoring by integrating more sensors. Sahoo and Rath, [19] proposed proposes a prototype model for location tracking using Global Positioning System (GPS) and Global System for Mobile Communication (GSM) technology. Overall system is partitioned into two major design units; Client Unit in Vehicle with Tracking Antenna and the Server Unit which GPS receiver and GSM modem is connected to PC. The client part which is the tracking device will be installed into the vehicle. The client unit is responsible for transmitting information wirelessly to Tracking Server located in the range. The server unit responds immediately after getting the message from the client, the system starts reading the current status of the vehicle and sends back the current speed and location to the mobile phone as a text message. The result shows that the object is being tracked with a minimal tracking error.

\section{CONCEPT OF THE PROPOSED SYSTEM}

The main component of this proposed system is a ballot box, a replica of the one used by the Independent National Electoral Commission (INEC)'s in Nigeria. This ballot box holds the ballot papers that are casted at each polling units. The results will be announced at each polling unit, thereafter, the result sheets, ballot boxes and other important materials are sealed in the box and transported to the appropriate electoral collation centre. The proposed system is divided into Tracking and Monitoring System. The pictorial diagram of the proposed is shown in figure 2 . The ballot box monitoring System is a software and hardware system enabling the position of ballot box to be tracked.The hardware design of the tracking unit of this system is shown schematically in figure 3. All the components needed for the system is assembled together in perfect order so as to ascertain the proposed tracking system. The system uses GPS to automatically track and record a box location. Activity is recorded by modules attached to each ballot box. Thereafter, the data is transmitted to a server and it is displayed on a central-monitoring computer through the customized web application system.

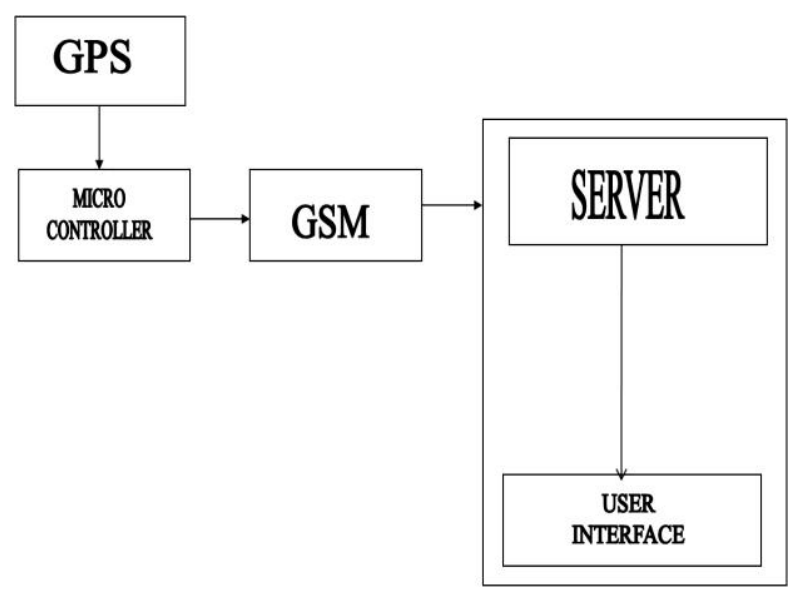

Fig 2: Block diagram of the Tracking \& Monitoring system

All the information sent by the tracking system that is placed in ballot box is received and stored in a database which is maintained by tracking server. This database has the feature which can be accessed by any authorized user using web interface. Therefore, authorized users can easily track the ballot box. To receive the information from the in-ballot box tracking unit, the tracking server must have an additional GSM receiver. The monitoring system unit mainly consists of GSM modem for effective communication with tracking system and web interface to provide user interface. Figure 4 illustrate the architecture of the monitoring server. 


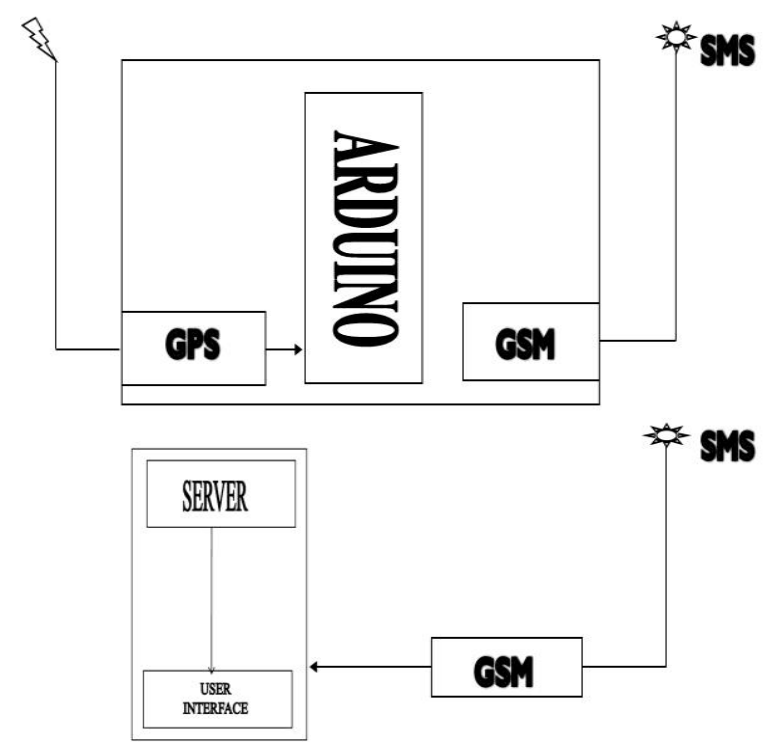

Fig3:Block diagram of the Proposed Tracking System

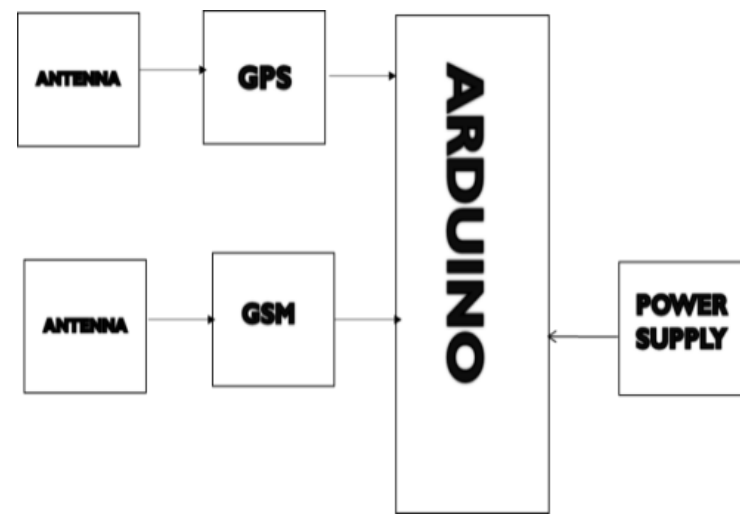

Fig. 4:System Architecture for the Monitoring System

\section{SYSTEM IMPLEMENTATION}

The monitoring station homepage is designed using Hypertext Markup Language (HTML), Java script and Hypertext Preprocessor language (PHP). The PHP code was used to extract the longitude and latitude of the ballot box from the txt file saved after each location request by the server. A new txt file is created intermittently and then the GPS tracker gets the location from the satellite and sends it through the send HTTP class in the Arduino code to the server. In the case the ballot box is snatched or taken away from the designated polling unit, it will be easily detected because of varying location position that will be received from the GPS by the server until it stops receiving the location points due to the separation from the server. The new location can then be forwarded to respective authorities by the time of investigation to establish the snatching allegation.

The system was implemented using a Google map integrated into a Web interface by using WAMP which served as a server and a micro-controller as the input device. After the development of the system and the configuration of each device, the system was then tested through the following. The Monitoring Station code is run on the Arduino IDE as the screenshot shown in figure 5, thereafter, the code is compiled and uploaded to the Seeeduino GPRS board as shown in figure 6 . The location of the ballot box is then displayed on server as figure 7 shown it after it has been implemented.

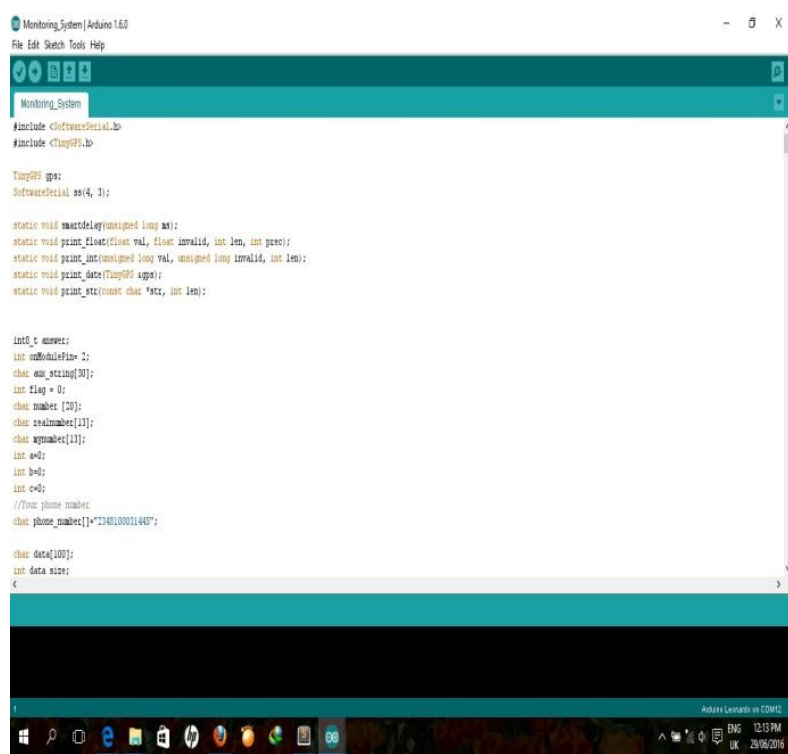

Fig 5: Screenshot of the Monitoring System code on the Arduino IDE

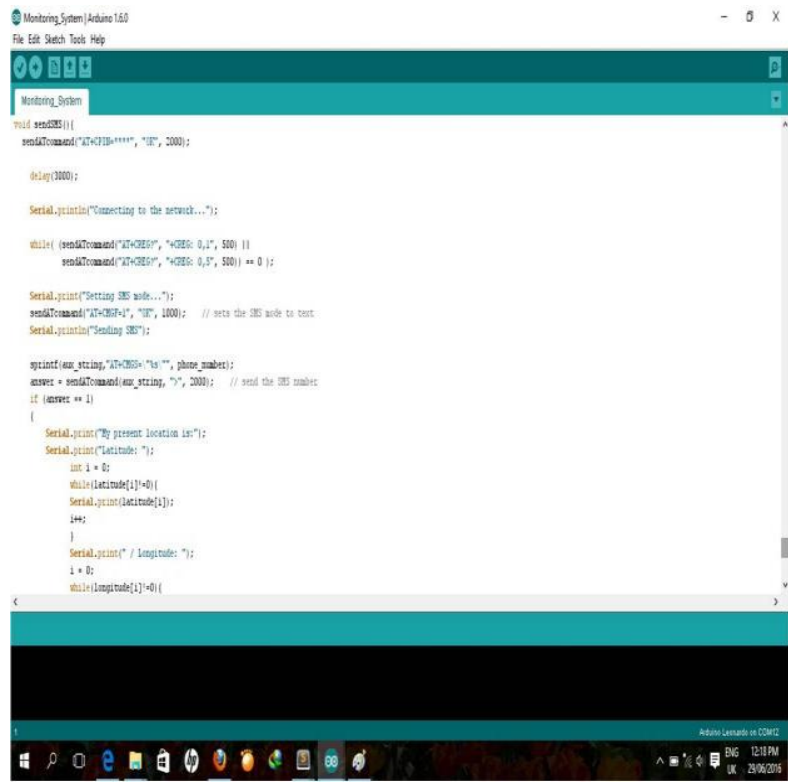

Fig 6: Monitoring System code compiled and uploaded on the Seeeduino GPRS board 


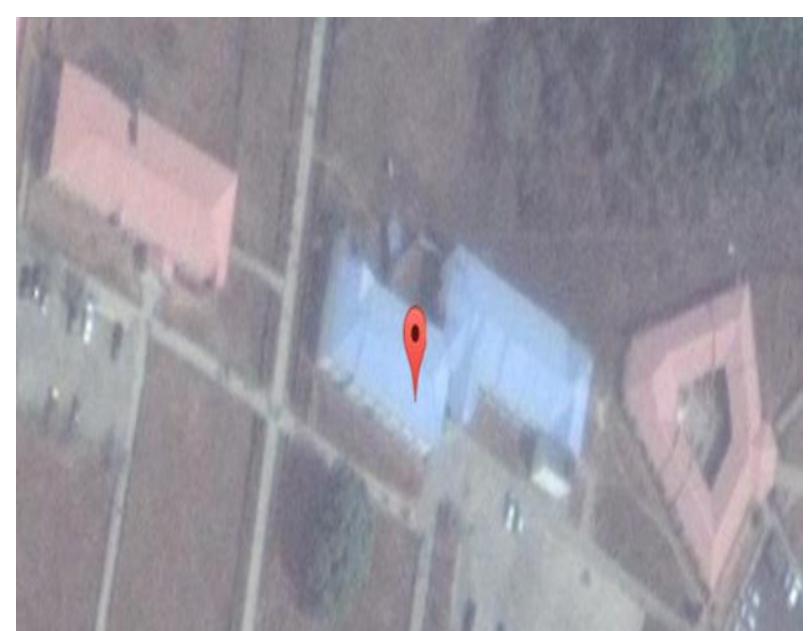

Fig. 7: Screenshot showing the location of the ballot box through the user interface

\section{CONCLUSION}

This work has demonstrated that GPS and GSM can be integrated with micro-controller to track and monitor the location of Ballot Boxes. However, the interface takes long to display the location on the map due to poor signal strength as a result of lower 2.5G GPRS sensors. In the future workvideo surveillance will be introduced to improve the system.

\section{REFERENCES}

[1] Mekki K., E. Bajic, and F. Chaxel (2019). ICT Express $5,1-7$.

[2] Chehri et al (2020). Framework of Optimizing the Deployment of IoT for PrecisionAgriculture Industry, Procedia Computer Science 176 , 2414-2422.

[3] https://www.rfidjournal.com/that-internet-of-things-thing

[4] Zheng D. E. and Carter W. A. (2015). Leveraging the Internet of Things for a More Efficient and Effective Military, A Report of the CSIS Strategic Technologies Program, Center for Strategic and International Studies, Washington, DC, ISBN: 978-1-4422-5890-7 (pb); 978-14422-5891-4 (eBook)

[5] World Bank, 2021. accessed on the $24^{\text {th }}$ September, 2021.

https://data.worldbank.org/indicator/SP.POP.TOTL?locat ions $=\mathrm{NG}$.

[6] Vanguard newspaper, (2020). Accessed on Sept 17, 2021, https://www.vanguardngr.com/2020/09/electoralmalpractice-3-varsity-dons-indicted-3-inec-officialssacked-in-aibom/.

[7] Card reader(2015). The Punch News.Archived from the original on 2 April 2015.Retrieved from Wikipedia, September 17, 2021. (https://web.archive.org/web/20150402112330/ http://www.punchng.com/news/we-support-inec-to-usecard-reader-us/)

[8] Gubbi J., R. Buyya, S. Marusic, M. Palaniswami, (2013). Internet of Things (IoT): a vi- sion, architectural elements, and future directions, Future Gener. Comput. Syst. 29, 1645-1660, doi: 10.1016/j.future.2013.01.010 .

[9] Miorandi D., S. Sicari, F. De Pellegrini, I. Chlamtac, (2012). Internet of things: Vision, applications and research challenges, Ad. Hoc.Netw. 10, 1497-1516, doi: 10.1016/j.adhoc.2012.02.016 .

[10] Lawal K. and H. N. Rafsanjani, (2021). Trends, benefits, risks, and challenges of IoT implementation in residential and commercial buildings, Energy and Built Environment, article in press.

[11] Vaishwanar P. (2014). Internet of Things, Aricent Marketing Research Report.

[12] Kuboye B. M. (2021). Comparative analysis of scheduling algorithms performance in a Long Term Evolution Network, Journal of Computer Science Research 3(4),Doi: https://doi.org/10.30564/jcsr.v3i4.3555.

[13] Sethi P. and S. R. Sarangi (2017). Internet of Things: Architectures, Protocols, and Applications. Journal of Electrical and Computer Engineering, Volume 2017, Article ID 9324035, 25 pages. https://doi.org/10.1155/2017/9324035

[14] Lee a, and K. Lee, (2015). The Internet of Things (IoT): Applications, investments, and challenges for enterprises https://dx.doi.org/10.1016/j.bushor.2015.03.008.

[15] Yugha R., S. Chithra, (2020). A survey on technologies and security protocols: refer- ence for future generation IoT, J. Netw. Comput. Appl. 169, 102763, doi: 10.1016/j.jnca.2020.102763.

[16] Satyanarayana, G., and Mazaruddin, S. (2013). Wireless Sensor Based Remote Monitoring System for Agriculture Using ZigBee and GPS. Conference on Advances in Communication and Control Systems pg. 110-114.

[17] Rathinakumar, R., and Manivannan, D. (2012). Wireless Accident Information System Using GSM and GPS. Research Journal of Applied Sciences, Engineering and Technology, pg. 3323-3326.

[18] Maurya, K., Singh, M., and Jain, N. (2012). Real Time Vehicle Tracking System using GSM and GPS Technology-An Anti-theft Tracking System. International Journal of Electronics and Computer Science Engineering. ISSN, 2277-1956.

[19] Sahoo, B., and Rath, S. (2013). Integrating GPS, GSM and cellular phone for location tracking and monitoring. 